\title{
Analytics for Measuring Library Use and Satisfaction of Mobile Apps
}

\author{
Simran Kaur \\ Senior Research Fellow, DLIS, Panjab University, Chandigarh, India \\ Email: phd1292017@gmail.com
}

Rupak Chakravarty

Professor, DLIS, Panjab University, Chandigarh, India

Email: rupak@pu.ac.in

\section{Library Hi Tech News \\ Emerald Publishing Limited \\ ISSN: 0741-9058}

DOI: $10.1108 /$ LHTN-04-2021-0014

Article Publication Date: 28 June 2021

Copyright $\odot$ 2021, Emerald Publishing Limited

\begin{abstract}
User review is a significant component of mobile app markets such as the Google Play Store, App Store, Microsoft Store and others. Users submit their reviews for downloaded apps on these sites in the form of star ratings and text reviews. Apps can contain huge volumes of feedback, making it difficult for the user and the developer to skim through thousands of such reviews to get an insight into usage and impact of such apps. Thus, the current study aims to assess the usage and satisfaction among users of the Mendeley's Android app vs iOS app.The analytics are performed by using Appbot analytics software which captured, monitored, measured and analyzed the review results for a particular period. Appbot provides easy-tounderstand insights of an app using artificial intelligence algorithm tools. The findings of the study reveal strong inclination, adoption and usage of Mendeley's Android app compared to that of iOS among users. The value of this research is in getting an insight of the pattern/behavior of users towards using apps on different platforms (Android vs iOS) and provides valuable results for the app developers in monitoring usage and enhancing features for the satisfaction of users. Without mobile app analytics, one will be blindly trying out different things without any evidence to back up their experiments.
\end{abstract}

\section{Keywords}

Mendeley, Mobile apps, Analytics, Appbot, Artificial intelligence, Smartphone, Android, iOS

\section{Introduction}

Mobile analytics help gain insight as to how consumers use an app, the app components they use most, and the tasks they perform. These experiences help create an action plan for further development, such as introducing and improving existing features and eliminating features of minimal or no use. Mobile games, for example, tend to concentrate on increasing sales with options for in-app purchases. Mobile analytics collects data to identify specific users, track their movements, record their behaviors and progress.

Mobile use surpassed desktop use in 2015 and smartphones are increasingly becoming the main Internet source for users. Consumers spend $70 \%$ of their screen time on mobile devices and apps. The smartphone penetration is expected to double from 468 million to 859 million in 2022 (https://www.assocham.org/newsdetail.php?id=7099). In the second quarter of 2019, 2.46 million and 
1.96 million apps were available in the leading Google Play Store and App Store respectively. It is projected that India will have 650 million Internet users by 2023

(https://www.statista.com/topics/2157/internet-usage-in-india/).Companies make use of analytics tools to achieve a competitive advantage in creating personalized mobile experiences. Data Statistics collected is important to marketing, distribution, and product management teams who use them to make informed decisions.

Hoon et al. (2013) evaluated 17,330 apps and 8.7 million reviews and found that users take the time to express their frustration by writing longer reviews, as compared to short reviews when they are not content with app uses. Ha and Wagner (2013) reviewed the feedback of Android users with Google Play and selected 60 free apps with 556 comments. After analysis, they found that a small subset of reviews focused on privacy and security, while most reviews concentrated on the quality of the apps. Khalid et al. (2015) identified the types of issues raised by users in mobile app reviews such as pricing, function, crashing, compatibility, privacy, network issue, response time, interface, and update issues. Vu et al. (2015) collected ratings and grouped keywords based on semantic similarity to gather and mine user opinions from app stores. A visualization tool of keywords helped to identify unusual trends. Park et al. (2015) built an AppLDA Subject Model for use on app descriptions and user reviews and helps developers recognize essential features of apps. Gu and Kim (2015) suggested a SUR-Miner, a description and categorization tool, and examined 2,000 sentences from 17 Google Play device reviews.

\section{Appbot}

Appbot is an analytics software that track, monitor and analyzes App reviews and ratings from Google Play, Apple (iOS and Mac), Windows Phone, Amazon (both apps and products) globally. Appbot can be helpful in understanding customers better. Generally, the product managers, customer analytics team, app marketers and development agencies use Appbot most heavily.

Features of Appbot include:

Sentiment Analysis, a subjective text mining and analysis tool, and uses AI to determine what users think about a specific app or product.

$\square$ Natural Language Processing feature of Appbot helps to understand and interpret unstructured text. The tool uses sentiment, keywords, common phrases, trending topics or tags and emotional analysis.

$\square$ Review Monitoring is performed via email alerts for apps and products with low review volumes. Appbot quantifies and monitors how many people make a particular request for functionality, or how many bug reports have been issued, and feeds the information back into the roadmap for improvement.

$\square$ RESTful API provides complete flexibility in monitoring and reporting app store review tracking. It aggregates reports for multiple apps, multi-series charts for several apps, reports comparing different data ranges, etc.

$\square \quad$ Integrations help close the consumer feedback loop by putting the customer's voice straight into the resources already in use by them. Appbot sends review data through Zapier to common messaging tools such as Slack, Zendesk, JIRA, Salesforce, etc.

\section{Appbot Example with the Mendeley Mobile Application}

Mendeley is a free bibliographic reference manager and academic social network released by Elsevier in 2008. Mendeley was monitored for different periods i.e. Mendeley Android app's data was captured from July 2015 since it first appeared on Google Play Store to March 2020. Similarly, the Mendeley iOS app was monitored from July 2013 as it first appeared on App Store to March 2020. Appbot software provides data results in categories such as Reviews, Sentiment, Words, Phrases, Topics and Languages. 
The Reviews tool demonstrates the statistics of a particular app's reviews for the chosen period. Filter pane helps to refine the results according to particular date range, keyword, star rating, sentiment, language, country, custom topic or tag, have or have not been replied to (Google Play only) and app version (iOS only). The Review Stats provides a summary as shown in figure 1

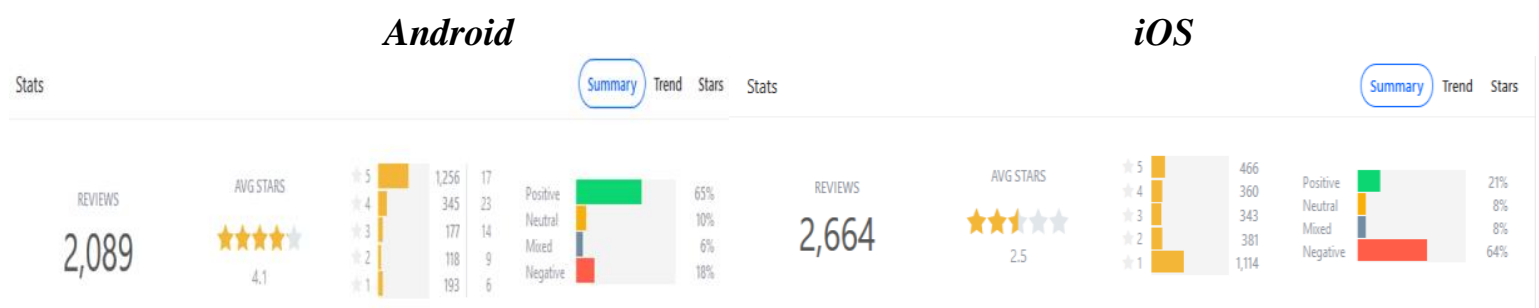

Figure 1. Reviews (Android vs iOS)

The Summary provides the total reviews received, an average rating of total reviews, star ratings (from 1 to 5) and sentiment analysis of a particular app on both Android and iOS. From Figure 1, it is inferred that the iOS platform (2664) received more reviews in text than Android (2089) over the time period and the average rating shows a negative trend in iOS than Android with 2.5 and 4.1 ratings respectively. Likewise, 1256 reviewers (65 percent) show positive feedback for the Android version as compared to just 466 reviewers (21 percent) for the iOS version.

Appbot's sentiment algorithm sorts app reviews and other data sources into 4 categories: positive, negative, neutral and mixed. The overall sentiment chart gives a measure of the average overall sentiments. Overall sentiments measure includes the volume of reviews, trends in review volume, star ratings of reviews, a trend in the star rating, the ratio of positive, neutral and negative reviews and its trend. The sentiment score is a score between $0-100 \%$, where $100 \%$ is the optimum sentiment (very positive). This tool showed that app users are optimally positive with an Android app with a 82\% sentiment score than an iOS app which received only a 50\% sentiment score. The Sentiment breakdown chart in figure 2 demonstrates the proportion of positive, negative, neutral and mixed sentiment for the selected reviews.

Android

iOS

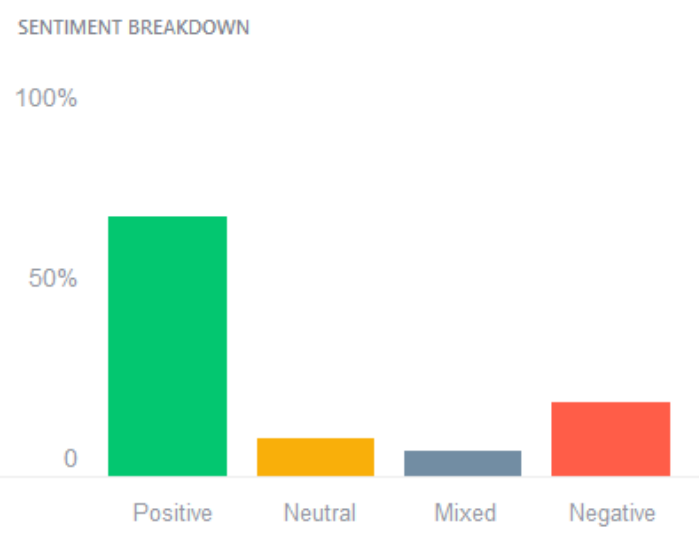

SENTIMENT BREAKDOWN

$100 \%$

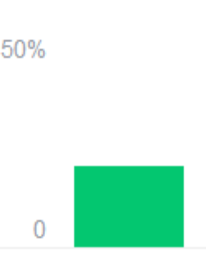

Positive

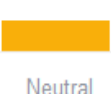

Neutral

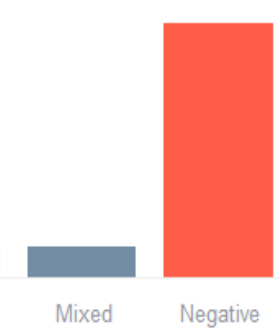

Figure 2. Sentiment Analysis (Android vs iOS) 
The Word tool analyzes all of the keywords in app reviews. It presents data of how frequently each word appears, patterns in their usage and a breakdown of the sentiment for a review containing that word. The word pattern has been categorized into six categories i.e. Interesting, Popular, Critical, Trending Up, Trending Down and New. The top ten words mostly covered up in the positive reviews thus signifies more satisfaction among users in both the Android and iOS version. In the iOS version for example, the top word 'iPad' appeared 434 times showing mostly in negative reviews with an overall review rate of $20.0 \%$ and trend. The top ten words mostly show negative sentiment and trend among users thus signifies less satisfaction for Mendeley iOS app among users.

AppBot also creates a word cloud that can be a visual tool for analyzing the sentiment for reviews containing specific words. The colors represent the sentiment of reviews containing each word, green for positive and red for negative. (see figure 3)

\section{Android}

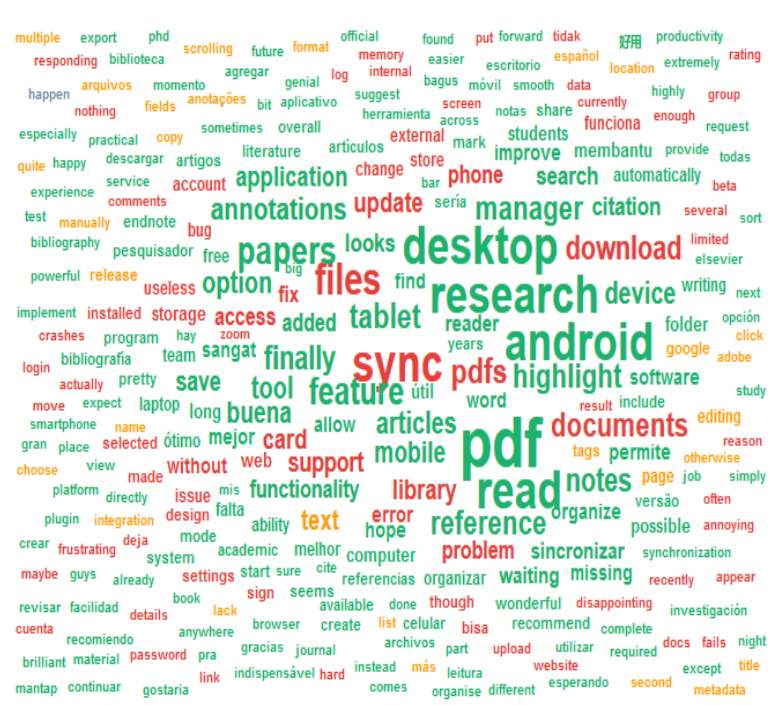

iOS

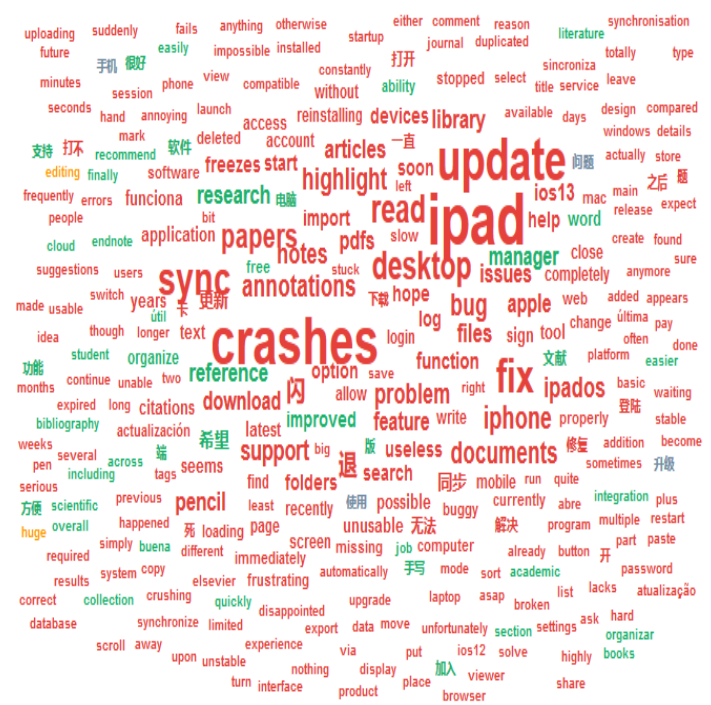

Figure 3. Word Cloud (Android vs iOS)

The Phrases tool analyzes all of the phrases in app reviews. It presents data that shows how frequently each phrase appears, patterns, and a breakdown of the sentiment for reviews containing that phrase. The phrases tool analysis examined the 2 phrases, 3 phrases, and 4 phrases appeared in review results. From figures for both the Android and iOS app results demonstrates the top five " 3 phrases" with its sentiment, mentions and mentions percentage. Further, Stats shows the individual results of each phrase. For example in an Android app, the 'Best Reference Manager' phrase appeared in total of five reviews with a $4.6 \%$ average star rating with $80 \%$ positive feedback by users. Similarly in the iOS app, 'Crashes every time' phrase shows $91 \%$ negative feedback by users with 1.7 average star ratin.

The Language tool allows to easily us to see commonly used languages in app reviews. In both platforms English is the top language used most by the users in reviews with 52.7\% (Android) and $70.8 \%$ (iOS) followed by others.

\section{Concluding Remarks}

Our example of the use of Appbot aimed to assess the usage and satisfaction of a mobile app, in this case Mendeley. The core of this study is based on the sentiment analysis of reviews in different categories. The findings of the overall study depict more significance of the Mendeley Android app than its iOS app although the features are almost the same in both platforms. Specifically, it is necessary 
to draw important conclusions when the negative emotions for the iOS app grow drastically in a given period. The reasons for negative sentiments could be compatibility issues or frequent bugs that appeared in the iOS app which needs to be taken into consideration by app developers and reviews their app timely so as to offer a robust and intuitive experience to users which will enrich their overall learning experience on the go anytime anywhere.

\section{References}

$\mathrm{Gu}, \mathrm{X}$. and Kim, S. (2015), "What parts of your apps are loved by users?", 30th IEEE/ACM International Conference on Automated Software Engineering, ASE 2015, IEEE Computer Society, pp. 760770, doi: 10.1109/ASE.2015.57.

Ha, E. and Wagner, D. (2013), "Do android users write about electric sheep? Examining consumer reviews in google play", 10th Annual IEEE CCNC- Mobile Device \& Platform \& Applications IEEE 10th Consumer Communications and Networking Conference (CCNC), IEEE, Las Vegas, pp. 149157, doi: 10.1109/cncc.2013.6488439.

Hoon, L., Vasa, R., Schneider, J.G. and Grundy, J. (2013), “An analysis of the mobile app review landscape: trends and implications", available at: https://pdfs.semanticscholar.org/2701/592f7d77a8a423218fe0b455918b5a4e0e2d.pdf (accessed 20 February 2020).

Khalid, H., Shihab, E., Nagappan, M. and Hassan, A.E. (2015), "What do mobile app users complain about?", IEEE Software, Vol. 32 No. 3, pp. 70-77, doi: 10.1109/MS.2014.50.

Park, D.H., Liu, M., Zhai, C. and Wang, H. (2015), “Leveraging user reviews to improve accuracy for mobile app retrieval", SIGIR 2015 - Proceedings of the 38th International ACM SIGIR Conference on Research and Development in Information Retrieval, Association for Computing Machinery, pp. 533-542, doi: 10.1145/2766462.2767759.

Vu, P.M., Nguyen, T.T., Pham, H.V. and Nguyen, T.T. (2015), "Mining user opinions in mobile app reviews: a keyword-based approach", 30th IEEE/ACM International Conference on Automated Software Engineering, IEEE, pp. 749-759, doi: 10.1109/ASE.2015.85.

\section{Further reading}

About Appbot (2020), available at: https://appbot.co/ (accessed 15 January 2020).

ElHady, H. (2018), "The ultimate guide to mobile app analytics", available at: https://instabug.com/blog/2018/04/22/ (accessed 10 February 2020).

Features of Appbot (2020), available at: https://appbot.co/features/ (accessed 31 January 2020).

Li, X., Zhang, B., Zhang, Z. and Stefanidis, K. (2020), "A sentiment-statistical approach for identifying problematic mobile app updates based on user reviews", Information, Vol. 11 No. 3, p. 152, doi: 10.3390/info11030152.

Wano, M. and Iio, J. (2014), "Relationship between reviews at app store and the categories for software", 2014 International Conference on Network-Based Information Systems, IEEE, pp. 580-583, doi: 10.1109/NBiS.2014.51.

\section{Citation}

Kaur, S. and Chakravarty, R. (2021), "Analytics for measuring library use and satisfaction of mobile apps", Library Hi Tech News, Vol. ahead-of-print No. ahead-of-

print. https://doi.org/10.1108/LHTN-04-2021-0014 